\title{
Systemic contact dermatitis caused by a surgical glue
}

\author{
Yue Wang ${ }^{1}$, Wenbin Liư ${ }^{2}$ Xiumin Yang ${ }^{2}$ \\ ${ }^{1}$ Department of Dermatology, Civil Aviation General Hospital, A-1 Gaojing, Changyang District, Beijing, China \\ 2Department of Dermatology, Beijing Tongren Hospital, Capital Medical University, Dongcheng District, Beijing, China \\ Adv Dermatol Allergol 2021; XXXVIII (2): 338-339 \\ DOI: https://doi.org/10.5114/ada.2021.106215
}

Surgical glues, such as octyl cyanoacrylate, are widely used by many surgeons as alternatives to traditional sutures. The polymerization of octyl cyanoacrylate monomers, which is catalysed by the presence of moisture on the surface of the polymer, occurs rapidly. Because of their convenience, rapid action and powerful clinical applications, surgical glues, represented by octyl cyanoacrylate, are strongly favoured by surgeons. Here, we report the first case of systemic contact dermatitis (SCD) caused by one type of surgical glue (SurgiSeal, Surgical Specialties Corporation, Virginia) and highlight the diagnosis of delayed-type hypersensitivity secondary to immediate-type hypersensitivity.

A healthy 38-year-old woman presented with poor wound healing and serous exudation on the dorsal side of her feet 2 weeks after Swanson implant (Wright Medical Technology, Arlington, Virginia) arthroplasty for Freiberg disease. SurgiSeal (Surgical Specialties Corporation, Virginia), a surgical glue containing octyl cyanoacrylate, was used to close the wound. Two weeks later, a diffuse, itchy rash began to appear covering her entire body. Locally, a wound infection was initially suspected, but cultures were negative. The patient had no history of the following: atopy; similar skin reactions; previous contact with artificial nail adhesives, false eyelash glues, medical devices with glucose sensors or other implants; or relevant dental treatments.

On examination, the wound was red with swelling and a small serous exudative scab; the long-standing erythematous maculopapular rash was restricted to this area (Figure 1). Scattered papules and papulovesicles were also observed on her trunk and limbs (Figure 2). Treatment with antihistamines and topical corticosteroids yielded poor results.

The patient underwent extensive patch testing with the European baseline series (Chemotechnique Diagnostics, Vellinge, Sweden), an acrylate series (not including octyl cyanoacrylate) and a plasticizer series. SurgiSeal and the silicone implant were also tested "as is". We used IQ Ultra chambers (Chemotechnique Diagnostics). Readings were taken on day (D) 3 and D5, according to the criteria of the International Contact Dermatitis Research Group (ICDRG) and European Society of Contact Dermatitis (ESCD) [1]. SurgiSeal caused positive reactions on both days (D3, +++; D5, $+++)$. Other allergens were negative, and so were tests for other surgery-related substances. Surgiseal was tested in 10 healthy volunteers, and none had any positive reaction. Thus, we considered her pruritic eruption to be delayed systemic allergic dermatitis triggered by SurgiSeal.

After debridement of the wound surface and removal of the SurgiSeal, the wound healed completely in 2 weeks, and the rashes involving her entire body responded gradually over the course of 3 weeks. A sample from her wound showed erosion, scabbing, and oedema of the epidermis, with prolonged and broadening trochanterellus. Focal inflammatory cells were observed throughout the dermis and septal panniculus, with elevated levels of lymphocytes and eosinophils. We followed up with the patient after 1 year and found that no relapse had occurred.

SCD is a skin condition in which an individual who is sensitized to an allergen via the cutaneous route will subsequently react to the same allergen via the systemic route [1]. SCD can manifest as pompholyx, cheilitis, generalized maculopapular-vesicular rash, erythema multiforme, vasculitis and urticaria [2]. The pathogenesis of SCD has been described as an immune-mediated, delayed- type hypersensitivity (type IV) reaction [3]. Following the first contact with an allergen, the allergen is then diffusely distributed into the skin and reacts with antigen-presenting cells that present the bound antigen to T lymphocytes. This results in the production of effector and memory T-cell populations, which are released into the blood circulation. Once these cells are sensitized, they return to the skin, ready to act on target cells when the individual is exposed to the allergen systemically, which leads to cutaneous manifestations. Although SCD has been described as a type IV delayed-type hypersensitivity, it may also involve a type III immune response as antigen-antibody complexes have been found in the skin and blood in such reactions [4]. Systemic exposure to an allergen can occur by uptake via cutaneous, trans-

Address for correspondence: Xiumin Yang MD, Department of Dermatology, Beijing Tongren Hospital, Capital Medical University, 1 Dongjiaominxiang St, Dongcheng District, Beijing 100730, China, phone/fax: +86 105826 8092, e-mail: YXM8092@163.com Received: 28.12.2018, accepted: 2.09.2019. 


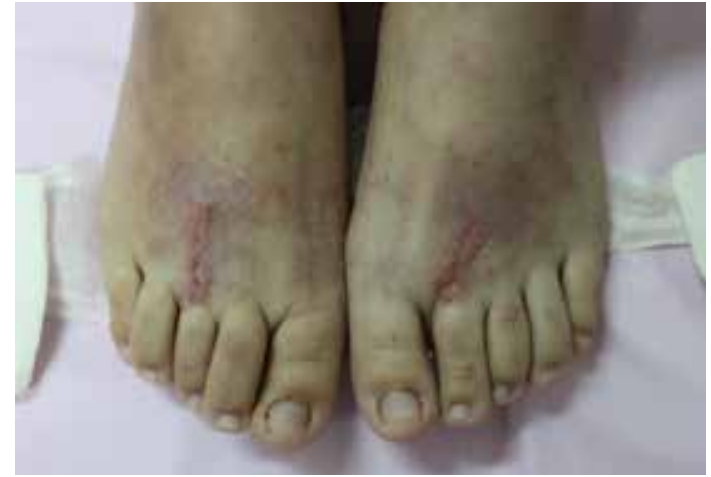

Figure 1. Redness and swelling around the wound with a small serous exudative scab and a long-standing erythematous maculopapular rash restricted to the wound

mucosal, oral, intravenous, intramuscular, and inhalational routes, as well as via implants [5]. In our case, the initial sensitization event is unknown, but it is likely to have been the exposure to the surgical glue. This previous exposure would explain why the early symptoms of contact dermatitis were restricted to the site of the surgical wound 2 weeks after silicone implant arthroplasty. Further systemic absorption of SurgiSeal, which resides and accumulates in tissues, could explain the generalized eruption that occurred on the patient's trunk and limbs.

SurgiSeal is a surgical glue that mainly contains octyl cyanoacrylate. Aquino et al. stated that when fully polymerized, (cyano)acrylates are non-immunogenic; however, if not completely polymerized, the monomers can be potent allergens [3]. Self-curing acrylates, such as octyl cyanoacrylate, typically contain substantially higher levels of residual monomers than acrylates cured by other processes [6]. For example, in the present case, in which SurgiSeal was applied to an open wound, the octyl cyanoacrylate remained in contact with the dermis and subcutaneous tissue for several weeks, which was sufficient to allow deep penetration and a great degree of accumulation in the surrounding tissue, thereby sensitizing the patient.

Although cyanoacrylates can cause irritant contact dermatitis [7], our patient experienced a delayed reaction at the wound site 2 weeks post exposure and a generalized eruption 4 weeks later. This pattern indicates a delayed-type hypersensitivity reaction rather than an elicitation reaction. Additionally, 10 healthy volunteers were tested for reactions to SurgiSeal, and none developed irritant contact dermatitis. Currently, we do not have access to the individual components of SurgiSeal for patch testing. However, excluding other possible factors of hypertrophic skin lesions and given the positive results of the patch testing with the surgical glue components, we consider octyl cyanoacrylate to be a reasonable candidate as the material responsible for our patient's SCD.

Because SurgiSeal is becoming increasingly popular as a standard means of wound closure, allergic reactions should be considered and recognized early, as SCD is an easily treat-

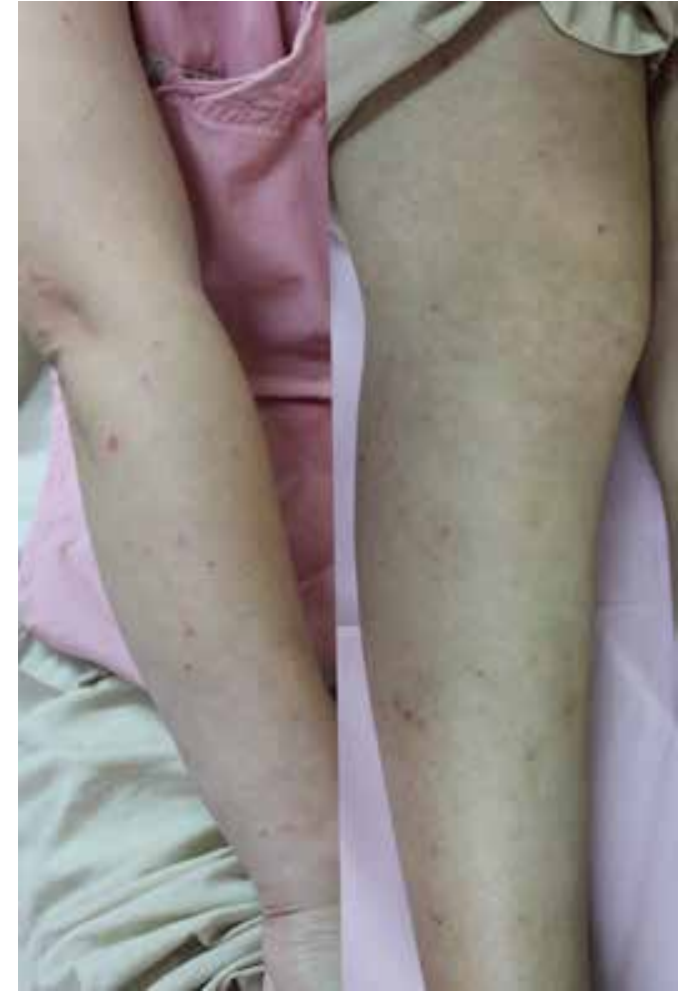

Figure 2. Scattered papules and papulovesicles on the limbs

able condition. All surgical applications, such as anaesthetics, antiseptics, antibiotics, implants and surgical glue, should be considered when patients present with diffuse pruritic eruptions.

\section{Conflict of interest}

The authors declare no conflict of interest.

\section{References}

1. Johansen JD, Aaltokorte K, Agner T, et al. European Society of Contact Dermatitis guideline for diagnostic patch testing recommendations on best practice. Contact Dermatitis 2015; 73: 195-221.

2. Bajaj AK, Saraswat A. Systemic contact dermatitis. Indian J Dermatol Venereol Leprol 2006; 72: 99-102.

3. Andersen KE. Systemic contact dermatitis. Int I Dermatol 2011; 50: 1445-56.

4. Lampel HP, Silvestri DL. Systemic contact dermatitis: current challenges and emerging treatments. Curr Treat Options Allergy 2014; 1: 348-57.

5. Aquino M, Mucci T. Systemic contact dermatitis and allergy to biomedical devices. Curr Allergy Asthma Rep 2013; 13: 518-27.

6. Maier T, Braun-Falco M, Hinz T, et al. Morphology of basal cell carcinoma in high definition optical coherence tomography: en-face and slice imaging mode, and comparison with histology. J Eur Acad Dermatol Venereol 2013; 27: e97-104.

7. Calnan CD. Cyanoacrylate dermatitis. Contact Dermatitis 1979; 5: 165-7. 